\title{
BIOTINIDASE DEFICIENCY SCREENING IN PATIENTS WITH GLOBAL DEVELOPMENTAL DELAY AT A TERTIARY CHILDREN'S HOSPITAL
}

M.O. Ogundele, Z. Bassi

Paediatrics, Alder Hey Royal Children's Foundation Hospital NHS Trust, Liverpool, UK

An evidence-based protocol for investigation of children with moderate/severe global developmental delay (GDD) was introduced in 2009, which recommended biotinidase deficiency (BD) screening early in the care pathway. We compared the rate and outcome of screening before and after the introduction of the guidelines.

We retrospectively analysed all patients tested for BD in a large children's hospital, three years before and one year after implementation of the guideline.

150 tests were requested in 138 patients aged 1 month to 17 years 7 mo (median 2 years 3 mo), with a total of $10(6.7 \%)$ abnormal results. The rate of tests requested (monthly per 1000 live births) increased from 0.31 in 2007 to 1.11 in 2010. 8 patients were diagnosed with partial BD corresponding to an annual incidence of 0.357 ( 0.18 to 0.54$)$ per 1000 live births, about 45 times higher than the expected estimated incidence of 0.008 ( 0.006 to 0.009$)$ in the general population.

The commonest symptoms were either neuro-developmental delay $(87.5 \%)$ or neurological abnormalities $(75 \%)$. The clinicians requesting the tests before and after the guideline implementation were the neurologists (54\% vs $19 \%)$ and community paediatricians (14\% vs $42 \%)$ respectively.

This study suggests that the prevalence of BD in a cohort of selected patients in a tertiary hospital with GDD is higher than in the general population. Until newborn screening is available in UK, it appears justifiable that children with neurological and developmental disorders should be tested for BD to enable early commencement of treatment and reversal of most symptoms. 